\title{
Understanding of Professional Ethics Among a Sample of Medical Practitioners in Bangladesh
}

\author{
Jahan MU' ${ }^{1}$, Rahman $\mathrm{SMM}^{2}$ \\ ${ }^{1}$ Bangladesh Medical Research Council (BMRC), Dhaka, Bangladesh; ${ }^{2}$ Academic Wing, Institute of \\ Public Health, Dhaka, Bangladesh
}

\begin{abstract}
Background: Ethical practice not only protects the rights of patients but is also a safeguard of medical practitioners. But, practicing medical ethics among the Bangladeshi health care professionals are not at a satisfactory level.
\end{abstract}

Objectives: The study was aimed to assess the level of understanding regarding medical ethics among the health care professionals.

Methods: A descriptive cross-sectional survey was conducted among registered 400 doctors of tertiary care hospitals and general practitioners in Dhaka City during June 2016 to January 2017. A non-probability sampling method was used for selecting the medical colleges, hospitals, institutions, medical university and general practitioners from the different areas of Dhaka city. A self-administered questionnaire was used for data collection assuring confidentiality and anonymity. Ethical approval was obtained from the National Research Ethics Committee (NREC) of Bangladesh Medical Research Council (BMRC) and from the Institutional Review Board (IRB) of the American University of Sovereign Nations (AUSN). The collected data were analysed through SPSS software, windows, version 21 (SPSS Inc., Chicago, IL, USA) and R programming language.

Results: Of the 400 respondents, $68.0 \%$ were male. Mean $( \pm \mathrm{SD})$ age of them was $30( \pm 6.5)$ years. About $93.0 \%$ respondents had only a MBBS degree and $65.0 \%$ were medical officers. About $38.0 \%$ respondents have below 5 years of clinical experience. Highest number of respondents got ethical information from book, second highest from lectures and then general text. About $32.0 \%$ respondents got ethical knowledge from one source. About $97.0 \%$ respondents agreed that medical ethics should be included in both undergraduate and postgraduate medical studies and $90.0 \%$ of them know only about theHippocratic Oath. Very few of them knew the Nuremberg Code (16.0\%) and Helsinki Declaration (16.0\%).Only $27.0 \%$ participants said that they never face any ethical problems in their clinical practice. About $75.0 \%$ reported that ethical practice is important in workplace. About $35.0 \%$ respondents have a very little knowledge and $46.0 \%$ have average knowledge on ethical law.

Conclusion: Findings of the study indicate that ethical knowledge of the respondents is not satisfactory although they realise that it is important in clinical practice and should include both undergraduate and graduate medical studies. Also need to develop practice in the workplace by law and legislations according to the guidelines of Bangladesh Medical \& Dental Council (BMDC) and Bangladesh Medical Association (BMA).

Keywords: Professional ethics, Medical practioners, Tertiary care hospitals, Hippocratic Oath

\section{Introduction}

Medical ethics also indicate the basic principles for medical professionals in order to practice of medicine and make a relationship with patients and health professionals. ${ }^{1}$ These health professionals practice this ethics in a compact environment. A few ethical issues like confidentiality, rapport with clients, and take consent for treatment have been faced by health professionals. ${ }^{2}$

*Correspondence: Dr. Mahmood-uz-jahan, Bangladesh Medical Research Council (BMRC),Dhaka, Bangladesh;e-mail: riponjahan@yahoo.com
Medical practice worldwide over and above the Hippocratic Oath is governed by normative or moral philosophical theories as enshrined in (contemporary) medical ethics. In developed countries; just as with governance, there are checks and balances which ensure good state governance, and in that sense good professional code of practice. In ancient civil society, medical ethics was applied according to Hippocratic Oath. With the change of time, codes of conduct, laws have been upgraded. ${ }^{3}$ Nowadays, medical ethics is a basic module of medical curriculum at the institutes of 
developed countries like USA, Canada, and many European countries. $^{4}$

Physicians and nurses commonly encounter ethical and legal issues in their workplace. However, many of these professionals are either unaware of their importance or unable to appropriately deal with these issues. Since the findings of the study, identify that learning at workplace has been valuable to gain knowledge about ethics and law, there is a need to identify those who appear to be indifferent to ethical and legal issues and devise means to sensitize them to these issues in the workplace. Practical education in ethics, particularly in a multidisciplinary setting, could assist in bridging the gap in ethical approaches between nurses and physicians. ${ }^{5}$

The issue of doctor-patient relationship is not that straight forward, as several factors influence the balance of forces at play in that relationship.

Doctors are very frequently come across ethical dilemmas in their day to day practice. They are not provided formal training in practical aspects of ethics in their medical curricula. In addition to this; identification of departmental lectures/teaching is also not very favourable among them, so there is an urgent need to include practical education of ethics, particularly in a multidisciplinary setting, to bridge the gap in the knowledge, attitude, and practices towards ethics in clinical practice and research. ${ }^{6}$

A study of ethical relations between physicians and pharmaceutical industries mentioned that existing Bangladesh Medical and Dental Council Actprohibit any unethical relation between physician and pharmaceutical industry; there are evidences that pharmaceutical industries are providing cash, hospitality, pleasure trips, medical equipments, books, sponsorship for conferences, workshop, decoration for chamber, professional and nonprofessional gifts for doctors. ${ }^{7}$

The Ministry of Health and Family Welfare of Bangladesh enforces and governs the medical practice according to legislation and laws. The government regulatory body also governs the hospitals, clinics, and diagnostic centers. Health professionals follow these principles to perform their professional tasks as well World Health Organization. ${ }^{8}$ There was given education about medical ethics in all medical institutes at the undergraduate level in Bangladesh. Professional misconduct and other fundamental issues were taught in that set course and curriculum for medical practice. ${ }^{9}$

In Bangladesh, the Bangladesh Medical and Dental Council (BMDC) regulate the discipline in relation to misconduct, malpractice, negligence, and conduct in medical practice. Regarding this issue, the investigator shall conduct study about assessment of knowledge among medical professionals towards medical ethics and challenges that hinder daily practice. So far there are very few of this type of research done in many countries. One of the research done in Indiabut in Bangladesh specifically this type of research not yet done. Moreover, from this research hope that if would be found out the factors for giving increased attention on medical ethics.

Ethical challenges are prevalent in modern-day medicine. Patients, their families, those who provide medical care, and the institutions where this care is conducted face difficult choices almost as a matter of routine. In addition to those arising in clinical practice, important and controversial ethical concerns also arise in the arena of clinical research and in educational practices. No domain of modern medicine is untouched. In particular, the problem of conflict of interest has become an issue especially relevant in this time. ${ }^{10}$

Health is a very important human value and hence healthcare is a paramount social good. In this context doctors have more responsibility on health of every people. ${ }^{11}$ The doctor-patient interaction and relationship has been considered as the core of medical practice.

In Bangladesh, there are approximately 3.05 physicians for 10,000 people, and in some hospitals, a single doctor must see the about 40-50 patients per day. The medical officers, post-graduate senior physicians, who after their office hour goes straight to respective private chamber or to some private hospital to work as a private practitioner. ${ }^{12}$

The theory of economy stated, like other individuals, doctors also aim to maximize their income, autonomy, professional ethics whereas theories of work motivation in Psychology give prominence to individual needs, values, personality and so on. In the context of developing countries, autonomy being a major reason to growth of private 
practice and as private practitioners are much independent, self-employed and can be entrepreneur themselves, scope of handsome income in contrast to the fixed salary of public service is also a motivating factor for the physicians to become more involved in the private practice. Nowadays, the private practice is very common and popular in Bangladesh but some physicians also faced problems in their private practice setting due to poor concern of ethics. In recent, the New Age reported on 6 July 2017 according to the Bangladesh Medical Association (BMA) based on 73 physicians came to be attacked on allegations that they had provided wrong treatment in the past five years. While the attack on medical service providers by patients or their relatives is condemnable, the BMA's decision to abstain from private practice to demand safe work environment is also a breach of their professional's ethics. ${ }^{13}$

\section{Materials and Methods}

This descriptive cross-sectional study was aimed to assess the level of understanding regarding medical ethics among health professionals. It was conducted among 400 registered medical practitioners working in the tertiary care hospitals as well as private general practitioners in Dhaka city. A nonprobability sampling method was chosen for selecting the study sites viz. Dhaka Medical colleges and hospitals, Sir Salimullah Medical College and Hospital Shahid Sorwadhary Medical College and Hospital, doing General Practice at Dhaka city.

A stratified random sampling technique was adopted to obtained the study population from different departments of the hospitals.

The study was conducted over a period of eight months from June 2016 to January 2017.

The sample size was estimated using the formula.

$$
\begin{aligned}
& \frac{Z^{2} p q}{d^{2}}=\frac{Z^{2} p(1-p)}{d^{2}} \\
&=\frac{1.962 \times 0.5 \times(1-0.5)}{0.05^{2}} \\
&=384
\end{aligned}
$$

Where,

$$
\mathrm{Z}=\text { Level of confidence or level of }
$$

significance $(95 \%)=1.96$

$$
\begin{aligned}
& \mathrm{p}=\text { Probability }(50 \%)=0.5 \\
& \mathrm{q}=(1-\mathrm{p})=(1-0.5)=0.5 \\
& \mathrm{~d}=\text { Acceptance error }=0.05
\end{aligned}
$$

Considering the non-response rate and missing data the sample size will be rounded to 400 .

After that, the purposive sampling technique was used for selecting health professionals.

\section{Data collection instrument}

Data were collected using a pre-tested, elfadministered questionnaires were filled anonymously after explanation to every participant. The data collectors briefed the objectives and benefits of this study to the respondents. All the study participants had been assured about their confidentiality and anonymity. In order to identify the questionnaires, numbers were assigned to each respondent (for future reference). Data collected from positive respondents only. Before starting data collection eight data collector were selected, all of them are young physicians who had just completed their graduation. They were given one week training on how to collect the data as well as how to prepare a concern form, how much time they spent and ethical implication of the study prior to collection of data.

All collected data were checked with full attention and sincere efforts and kept in the sequences in which those have been numbered. The data were entered into personal computers by expert computer operator followed to SPSS programme (version 23.0). The appropriate statistical tests were done depending on the distribution of the data. Both descriptive and inferential statistics were used and presented in the form of tables, graphs and diagrams.

The investigator was fully monitored whole data collection process. After data collection, firstly checking, editing and omission were done by investigator on the scientific basis. Data analysis was done by SPSS 23.0 Version, R programming language and Ms-Excel software.

Ethical clearance was obtained from the National Research Ethics Committee (NREC) of Bangladesh Medical Research Council. The protocol had been peer reviewed by the authority of National Research Ethics Committee. Before submitting to the NREC, protocol also reviewed by the American University of Sovereign Nations (AUSN) IRB.

Due to resource constraints, the study was only conducted in the Dhaka City among some selected medical colleges, hospitals and general practitioners 
with small number of sample size (only 400 doctors) which make barriers to generalise the study findings.

\section{Results}

This descriptive cross sectional study was carried out among 400 doctors. Of them, 68.0\% respondents were male and $32.0 \%$ female.

Experience, knowledge as well as basic perceptions, all components depend on age. For this aspect, the study included the participants age. Out of 400 respondents 262 were in 21-30 years in age group and 120 were 31-40 years in group and very few (only 18) was in above age group (figure 1). The mean age $\pm \mathrm{SD}=30.37 \pm 6.454$ years of the study participants.

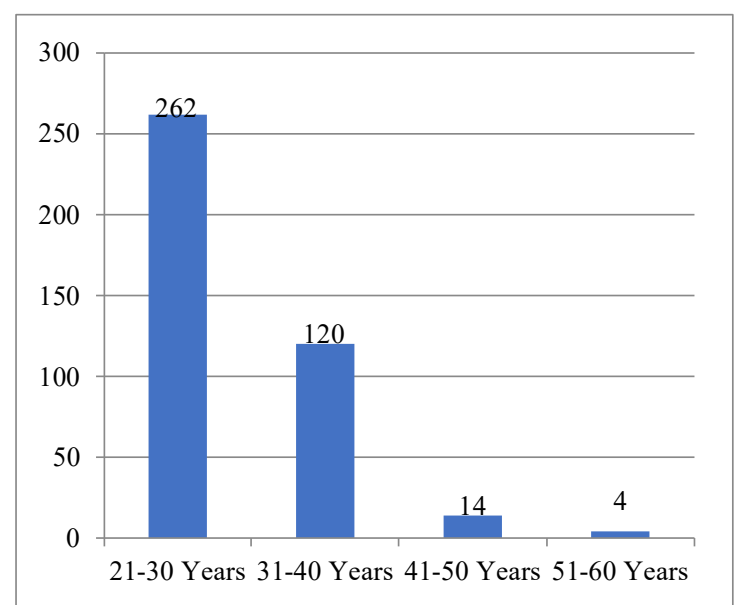

Figure 1: Distribution of the respondents according to their age group

The study found major group (238 respondents) was the medical officer. that the second most study participants about 77 were postgraduate students and third general practitioner. Only twenty onewere Consultants (figure 2).

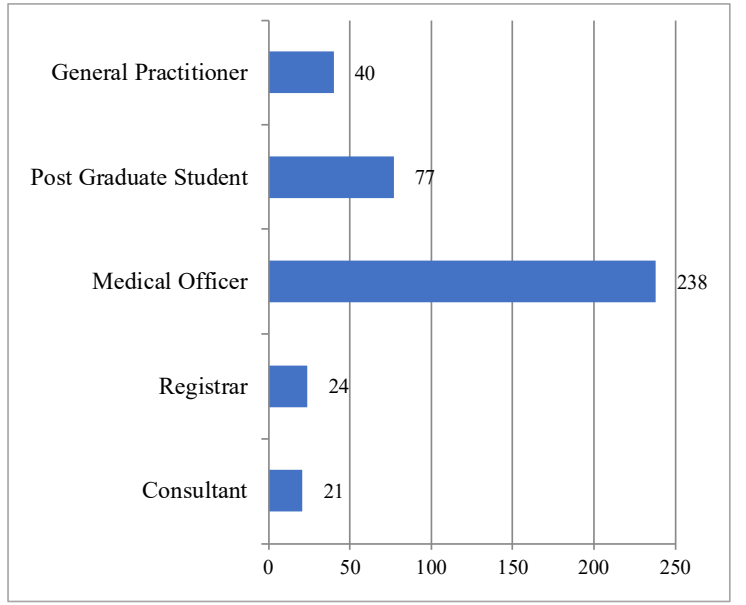

Figure 2: Distribution of the respondents according to their designation
The study enrolled 400 medical doctors whereas majority (372 respondents) $93.0 \%$ were MBBS and rest of them (28 respondents) only $7.0 \%$ were $\mathrm{PhD}$, MD/MS, FCPS, MPH and Diploma holders (figure 3).

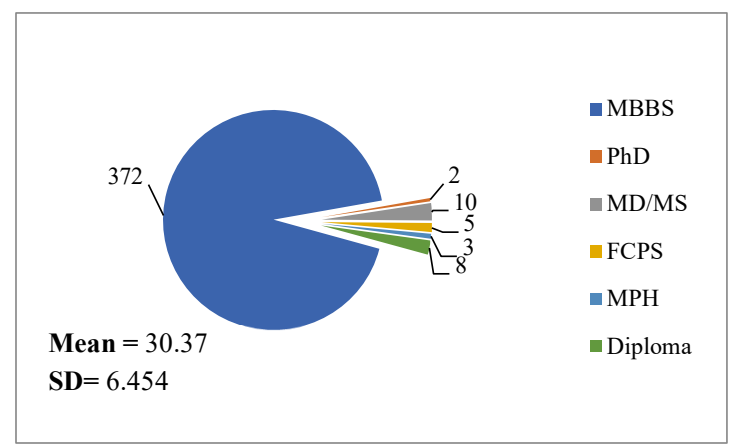

Figure 3: Distribution of the respondents according to their height level of Education

Among the respondents, the majority $(38.0 \%)$ had working experience of below 5 years, $25.0 \%$ had 6 10 years working experience, $28.0 \%$ had $11-15$ years and the rest of them, $9 \%$, were more than 15 years working experience (table I).

Table I: Distribution of the respondents according to their work experience

\begin{tabular}{lcc}
\hline Years of Experience & Frequency & Percentage (\%) \\
\hline Below 5 years & 153 & 38.3 \\
6-10 Years & 101 & 25.3 \\
11-15 years & 111 & 27.8 \\
16-20 years & 11 & 2.8 \\
21-25 years & 21 & 5.3 \\
above 25 years & 3 & 0.8 \\
Total & $\mathbf{4 0 0}$ & $\mathbf{1 0 0 . 0}$ \\
\hline
\end{tabular}

The majority of the participants gained knowledge from books on ethics, lectures, general texts, seminar and workshop, clinical training and ethics journal. The data may present that all sources are more helpful to gather knowledge on medical ethics (table II).

Table II: Distribution of the respondents according to their gained knowledge on medical ethics from different sources

\begin{tabular}{lccc}
\hline Sources of ethics & Yes & No & Total \\
\hline Ethics iournals & 109 & 291 & \\
Books on ethics & 245 & 155 & \\
General texts & 177 & 223 & \\
Media (Newspapers / TV) & 97 & 303 & \\
Seminar and Workshops & 151 & 249 & 400 \\
Lectures (UG / CME) & 182 & 218 & \\
Panel discussions & 23 & 377 & \\
Case conferences & 54 & 346 & \\
Clinical Training & 131 & 269 & \\
Others & 13 & 387 & \\
\hline
\end{tabular}


After calculation of data, $32.0 \%$ respondents informed they learned from one source, $16.3 \%$ learnt from three sources, $16.0 \%$ learnt from two sources, more than six sources was $1.8 \%$ (table III)

Table III: Usefulness of the gained knowledge on medical ethics from different sources

\begin{tabular}{ccc}
\hline No. of sources & Frequency & Percent \\
\hline Zero & 32 & 8.0 \\
One & 128 & 32.0 \\
Two & 64 & 16.0 \\
Three & 65 & 16.3 \\
Four & 42 & 10.5 \\
Five & 34 & 8.5 \\
Six & 28 & 7.0 \\
Seven & 4 & 1.0 \\
Nine & 3 & .8 \\
Total & $\mathbf{4 0 0}$ & $\mathbf{1 0 0 . 0}$
\end{tabular}

There was a significant association between importance of knowledge on ethics in work place and usefulness source of knowledge on medical ethics (table IV).

The study dealt with the views of participants regarding inclusion of medical ethics in curriculum (undergraduate and post-graduate) and found that $97.0 \%$ respondents ( 388 persons) said that medical ethics should be included in the curriculum.

Table IV: Relationship between importance of medical ethics knowledge related with its number of sources.

\begin{tabular}{|c|c|c|c|c|c|c|c|c|c|c|}
\hline \multicolumn{11}{|c|}{ Number of Sources } \\
\hline Importance: & Zero & One & Two & Three & Four & Five & Six & Seven & Nine & Total \\
\hline Not at all & 0 & 2 & 0 & 1 & 0 & 0 & 0 & 0 & 0 & 3 \\
\hline $\begin{array}{l}\text { Somewhat } \\
\text { Important }\end{array}$ & 0 & 8 & 1 & 2 & 0 & 0 & 0 & 0 & 0 & 11 \\
\hline Important & 3 & 32 & 10 & 9 & 7 & 3 & 1 & 0 & 0 & 65 \\
\hline Very Important & 8 & 54 & 22 & 31 & 25 & 20 & 10 & 2 & 3 & 175 \\
\hline $\begin{array}{l}\text { Extremely } \\
\text { important }\end{array}$ & 21 & 32 & 31 & 22 & 10 & 11 & 17 & 2 & 0 & 146 \\
\hline Total & 32 & 128 & 64 & 65 & 42 & 34 & 28 & 4 & 3 & 400 \\
\hline
\end{tabular}

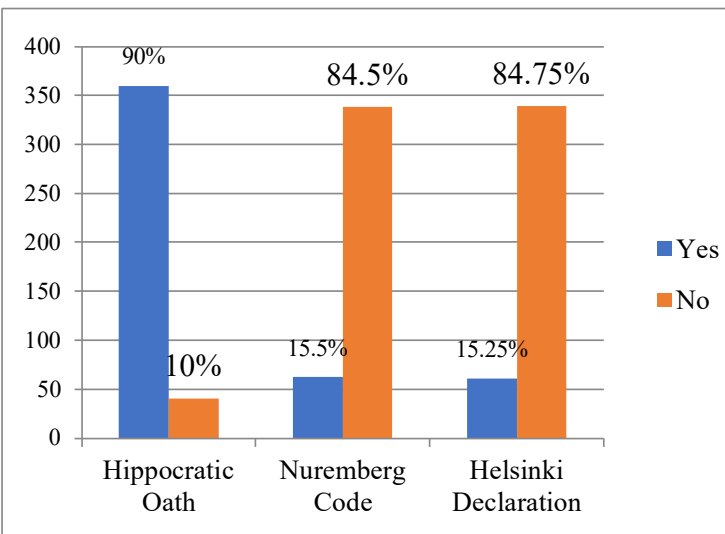

Figure 4: Distribution of the respondents according to the main content of ethics

The ethics has a standard guideline which is highly accepted worldwide. There are some contents of ethics among the doctors are very popular in Bangladesh (such as Hippocratic Oath, Nuremberg Code, Helsinki Declaration). To consider the contents, the researchers were interviewed to the participants to know about those. The participants respond by cluster barwhich shows that majority $(90.0 \%)$ have knowledge about the Hippocratic Oath (figure 4). On the other hand, only $15.0 \%$ of respondents have very few knowledge on Nuremberg Code and Helsinki Declaration.
It was to be found that $27.0 \%$ respondents said that they never faces any difficulties in their working place. But other respondents, $20.5 \%$ faced a problem monthly, $19.0 \%$ daily, $16.8 \%$ weekly and $16.8 \%$ yearly or less (table V).

Table V: Distribution of the respondents according to their status of facing difficulties in work place regarding ethics and its importance.

\begin{tabular}{lcc}
\hline \multicolumn{1}{c}{ Facing difficulties } & Frequency & Percent \\
\hline Never & 108 & 27.0 \\
Daily & 76 & 19.0 \\
Weekly & 67 & 16.8 \\
Monthly & 82 & 20.5 \\
Yearly or less & 67 & 16.8 \\
& & \\
\multicolumn{1}{c}{ Importance of Knowledge on ethics } \\
Not at all & 10 & 2.5 \\
A little & 33 & 8.3 \\
Moderately & 54 & 13.5 \\
Very important & 301 & 75.3 \\
Not sure & 2 & .5 \\
Total & $\mathbf{4 0 0}$ & $\mathbf{1 0 0 . 0}$ \\
\hline
\end{tabular}

On the other hand, it had been observed that $75.3 \%$ of the respondents opinion regarding importance of 
ethics practice in workplace were expressed as 'very important'.

Table VI: Participants knowledge on law pertaining at work place

\begin{tabular}{lcc}
\multicolumn{1}{c}{ place } & & \\
\hline Not at all & Frequency & Percent \\
a little & 17 & 4.3 \\
Average & 141 & 35.3 \\
Most of them & 187 & 46.8 \\
Not sure & 41 & 10.3 \\
Total & 14 & 3.5 \\
\hline
\end{tabular}

\section{Discussion}

Application of medical ethics in clinical practice not only ensures the patients' right but also responsible for securing clinical practice of the health profession. This study tries to explore the knowledge and practice of medical ethics among the doctors in Bangladesh.

In terms of clinical experience, more than half $(52.0 \%)$ respondents had 6 to 15 years experiences and one third had less than 5 years experiences. Research studies found that the senior doctors (either by age or qualification) had better knowledge of healthcare ethics either because of experience or increased attendance at conferences and workshops. Similar findings had also been raveled by an Indian study and a study from the West Indies. ${ }^{14,15}$

Study revealed that book on ethics $(61.0 \%)$, lectures $(45.0 \%)$, general texts $(44.0 \%)$ and seminar/workshop $(38.0 \%)$ were the more common source of ethical knowledge of the respondents. Additionally about one third respondents $(32.0 \%)$ learn ethical knowledge from only one source and very minimal proportion learn from more than one sources.

In one sense this is not a good practice for health professionals just to depend on books and class lectures in this modern world.

Majority of the respondents $(97.0 \%)$ were agreeing with the inclusion of Medical ethics in the undergraduate and post graduate curriculum. This indicates that now they are realizing the importance of medical ethics. Center for medical education should consider this issue and keep medical ethics is curriculum as a priority basis. Another literature suggested that the proper ethics practice is the very crucial component for doctors in their working place because it reduce the anticipating problems and helps to avoiding conflicts of interest, balancing profit with serving patients and providing charity care, equally justify the patients, no raise any discrimination (such as poor vs rich) through other influential people, Managing the vulnerable patients who may not have decision-making capacity, providing care with minimal benefit. ${ }^{16}$

In order to consider medical ethics in clinical practice one need to know about the ethics by him/herself. But in Bangladesh the situation is poor. Although most $(90.0 \%)$ of the respondents are familiar of Hippocratic Oath and very few (15.0\%) have knowledge on Nuremberg code and Helsinki declaration. According to the Bangladesh Medical and Dental Council (BMDC), only 16 hours (6 hours lectures and 10 hours tutorial) was assigned for the Behavioral Science (attention to Doctor-Patient Relationship) under the subject of Community Medicine which is very short to cover the ethics information elaborately. Literature explored that historically the oath has been used to swear in a student at the beginning of medical students. This oath is still used in modern times but has been updated to fit with the different language and culture. One of the most important ideas codified in the Hippocratic oath is that the physician is accountable for his/her actions should problems arise and the oath has an extremely individualistic perspective. ${ }^{17}$ However, this reflection has found from the study itself that less than half $(47.0 \%)$ respondents have average knowledge on law pertaining at workplace. The reason for this situation is lack of mandate from government and Bangladesh Medical and Dental Council (BMDC) following ethical practice according in clinical practice.

Not obeying the ethical practice may constitute several problems including practitioner himself. Although, practitioners in Bangladesh don't need to face problems regarding ethical issue in their clinical practice as most of the clients are not well educated but day by day the situation is changing. As this study reflects the same situation like $27.0 \%$ never face any ethical related problem in their practice life but rest of them faced problem at least yearly to at most daily. Therefore, more than two third $(75.0 \%)$ respondents are now agreeing with the importance of medical ethics. But unfortunately, less than half $(46.0 \%)$ have average knowledge 
about law in the workplace. A study revealed that in India, legal suits on doctors have increased in recent year; this can be largely attributed to lack of knowledge and practice of medical ethics. ${ }^{18}$ So, necessary steps should be taken to improve the knowledge on law in the workplace of the practitioners. Bangladesh Medical and Dental Council (BMDC) and Bangladesh Medical Association (BMA) can arrange several seminar and workshop throughout the country on a regular basis. Educational leaflet can be distributed among the practitioners. Mobile text can be one of the better options for regular update information.

\section{Conclusion}

Findings of this study shows that the current knowledge level and practice of medical ethics among the health practitioners in Bangladesh. Are not at satisfactory level. Responsible authorities like BMDC and professional body like BMA should think about this matter. Inclusion of medical ethics elaborately in the undergraduate and postgraduate medial courses which enrich the medical professionals to practice the ethics properly, thus recommended.

\section{Conflict of Interest: None}

Funding: No funding for this study

Ethical approval: National Research Ethics Committee (NREC) of Bangladesh Medical Research Council, Dhaka.

Submitted: $27^{\text {th }}$ August 2020

Final revision received: $17^{\text {th }}$ November 2020

Accepted: $25^{\text {th }}$ November 2020

Published: $1^{\text {st }}$ December, 2020

\section{References}

1. Talukder MHK, Nazneen R, Hossain MZ, Ishrat JC. 'Review article: Basic ideas on medical ethics. Bangladesh Journal of Medical Science. 2010; 9: 131-35.

2. Greenwood B. Legal and Ethical Issues that Health Care Professionals Face', [Internet]. [Place unknown]. Accessed 12 December, 2016, URL: www.work.chron.com/legal-ethical-issueshealth-care-professionals-face $5648 . \mathrm{html}$.

3. Sauer JE. Ethical problems facing the industry.Hospital and Health Services Administration. 1985; 30: 44-53.
4. Batistatou A, Doulis EA, Tiniakos D, Anogiannaki A, Charalabopoulos K. The introduction of medical humanities in the undergraduate curriculum of Greek medical schools: Challenge and necessity.Hippokratia.2010;14:241-3.

5. Walrond ER, Jonnalagadda R, Hariharan $S$, Moseley HSL. Knowledge and Practice of Medical Students at the Cave Hill Campus in Relation to Ethics and Law in Healthcare.West Indian Med J. 2006; 55: 42-47.

6. Mohammad M, Ahmad F, Rahman SZ, Gupta V, Salman T. Knowledge, Attitudes and Practices of Bioethics among Doctors in a Tertiary Care Government Teaching Hospital in India.J Clinic Res Bioeth. 2011;.2:2-6.

7. Bangladesh Medical and Dental Council (BMDC) Act 2010, Accessed 12 July, 2017, URL:www.clcbd.org/document/855.html.

8. World Health Organization. Regional Office for South-East Asia: Health ethics in six SEAR countries. New Delhi, 1996.

9. Bangladesh Medical and Dental Council 2012, 'Bangladesh: Curriculum for Under-graduate Medical Education in Bangladesh', Accessed 09 July 2017,

URL:www.mmc.gov.bd/downloada ble\%20file/new_curriculum/The\%20updated $\% 2$ 0MBBS\%20Curriculum\% 202012.pdf.

10. MacKenzie RC. What Would a Good Doctor Do? Reflections on the Ethics of Medicine.HSS J. 2009; 5:196-199.

11. Hadzibegovic I, Danic A, Hren D.Medical Students' opinion on Physicians' strike: Cross sectional questionnaire study. Croat Med J. 2004; 45: 63-66.

12. Bangladesh Health System Review 2015. Health Systems in Transition, vol. 5, no. 3, pp.1-48. Accessed10 July, 2017 URL:www.wpro.who.int/asia_pacific_observator y/hits/series/bgd_health_system_review.pdf.

13. The New Age 2017, Dhaka. 'Physicians continue to compromise medical ethics', published-20 Jun 2017, Accessed 12 July 2017, URL:www.newagebd.net/article/18143/physicia ns-continue-to-compromise-medical-ethics.

14. Brogen SA, Rajkumari B, Laishram J, JoyA. 2009, 'Knowledge and attitudes of doctors on 
medical ethics in a teaching hospital, Manipur.Indian Journal of Medical Ethics. 2009; 6:194-97.

15. Seetharaman H, Ramesh J, Errol W, HarleyM. 2006, Knowledge, attitudes and practice of healthcare ethics and law among doctors and nurses in Barbados.BMC Medical Ethics. 2006; 7:1-9.

16. Kreimer S. 'Five Ethical Challenges in Healthcare', AMN Healthcare, Inc. 2010.
Accessed 11 July, 2017,

URL:www.amnhealthcare.com/latest-healthcarenews/five-ethical-challenges-healthcare.

17. The Hippocratic Oath 2017. 'The Evolution of Medical Ethics'.Accessed 8 July 2017, URL:www.owlspace-ccm.rice.edu/access/content/

18. Joga Rao SV. 2009, 'Medical negligence liability under the consumer protection act: A review of judicial perspective.Indian J Urol.2009; 25:361-71. 